\title{
Macrophage and dendritic cell infiltration in head and neck squamous-cell carcinoma; an immunohistochemical study
}

\author{
Jeroen D. Kerrebijn ${ }^{1}$, Alphons J. M. Balm², Paul P. Knegt ${ }^{1}$, Cees A. Meeuwis ${ }^{3}$, Hemmo A. Drexhage ${ }^{4}$ \\ ${ }^{1}$ Department of Otolaryngology, University Hospital Dijkzigt, Rotterdam, The Netherlands \\ 2 Department of Otolaryngology/Head and Neck Surgery, The Netherlands Cancer Institute (Antonie van Leeuwenhoekhuis), Amsterdam, \\ The Netherlands \\ ${ }^{3}$ Department of Otolaryngology, Dr. Daniel den Hoed Cancer Clinic, Rotterdam, The Netherlands \\ ${ }^{4}$ Department of Immunology, Erasmus University, Rotterdam, The Netherlands
}

Received: 14 July 1993/Accepted: 20 September 1993

\begin{abstract}
A study was undertaken to help us reach a better understanding of the tumor-infiltrating pattern of lymphoid cells and in particular of monocyte-derived cells, namely the $\mathrm{CD}^{2} 8^{+}$, acid-phosphatase-expressing scavenger macrophages and the MHC-class-II- and S100-antigen-presenting dendritic cells in head and neck squamous-cell carcinoma. In the stroma of the tumors distinctive small fields of lymphocytes were found, the T cell areas of these fields being intermingled with dendritic cells. Intra-epithelial dendritic cell infiltration was low. The infiltrative pattern of macrophages was similar to patterns described in earlier studies with substantial stromal invasion and inconsistent intra-epithelial invasion, but small granuloma-like structures of $\mathrm{CD} 8^{+}$macrophage-like cells, found in the stroma of tumors, have not been reported before. The histochemical localization of the tumor-infiltrated dendritic cells and macrophages supports the view that the former cells are involved in the sensitization to tumor antigens, whereas the latter cells are involved in tumor cytotoxicity/scavenging of tumor cell debris. Although it has been shown in the past that transmembranal (TM) factors (p15E-like factors) present in the serum and tumor of patients with cancer of the head and neck have suppressive effects on monocyte/macrophage/dendritic cell function, a relationship between the intensity of epithelial staining for TM factors and the infiltrative pattern of monocytes/macrophages/dendritic cells could not be demonstrated.
\end{abstract}

Key words: Macrophages - Dendritic cells - Head and neck cancer - Squamous-cell carcinoma

\section{Introduction}

There is experimental evidence that the cell-mediated immune system plays an important role in the defence against

Correspondence to: J. D. Kerrebijn, Dept. of Otolaryngology/Head and Neck Surgery, University Hospital Dijkzigt, Dr. Molewaterplein 40, 3015 GD Rotterdam, The Netherlands head and neck squamous carcinoma cells [4, 8, 9, 15, 22]. Cell-mediated immune functions are executed by T lymphocytes, monocytes, macrophages and dendritic cells. It is therefore of relevance that defects in the function of these cells have been described in patients with head and neck cancer.

Dendritic cells play a role in tumor defence by presenting tumor-associated antigens to the immune system. Dendritic cells are monocyte-derived cells, with a distinctive morphology and a characteristic marker pattern in immunohistology. The cell shows long cytoplasmic veils or dendrites, has a kidney-shaped nucleus and usually reacts strongly with MHC-class-П-directed antibodies amongst which are L25 and RFD1. The cell is also often positive for the protein S100. Dendritic cells have a weak expression (sometimes only in the form of a paranuclear spot) of CD68, CD14 and of the enzyme acid phosphatase. To execute its antigen-presenting function, the dendritic cell forms clusters with lymphocytes and it is therefore of importance that, in patients with head and neck cancer, the capability of blood dendritic cells to form clusters with allogenic lymphocytes has been described as impaired [19].

Macrophages are also monocyte-derived cells and mainly have a killer and scavenger function. Macrophages are strongly positive for CD68, and the enzyme acid phosphatase; occasionally, they are positive for MHC class II markers. Morphologically, they are large, rounded cells lacking long cytoplasmic protrusions. Defects in the tumoricidal capacity of macrophages have also been reported in patients with head and neck cancer [4].

The majority of reports on the infiltration of lymphoid cells into head and neck tumors deal with the infiltration of lymphocytes. Reports on the pattern of infiltration of monocytes, macrophages and dendritic cells are scarce. It has been described that patients with a dense infiltration of S100-positive dendritic cells in nasopharyngeal carcinoma are likely to survive longer than those without [16]. No such correlation appears to exist, however, between the density of lysosome-positive macrophage infiltration in or around tumor nests and the prognosis or stage of the tumor [16]. 
Table 1. Monoclonal antibodies

\begin{tabular}{lllll}
\hline Epitope & Antibody & Dilution & Specificity & Source \\
\hline RFD1 & & $1: 1000$ & $\begin{array}{l}\text { Active dendritic cells and subset of B cells } \\
\text { (class-II-MHC-associated antigen) }\end{array}$ & Kindly provided by L. Poulter, London \\
L25 & & $1: 1000$ & $\begin{array}{l}\text { Dendritic cells and B cells } \\
\text { Dendritic cells (mainly intra-epithelial) }\end{array}$ & Kindly provided by Ishii, Sapporo \\
S100 & Ki-M7 & $1: 500$ & Macrophages (strong cytoplasmic expression) & Dakopatts, Glostrup \\
CD68 & LeuM3 & $1: 200$ & Monocytes, macrophages & Becton Dickinson, San Jose, Calif. \\
CD14 & Leu4 & $1: 600$ & Immature and mature functional T cells & Becton Dickinson, San Jose, Calif. \\
CD3 & CLB-B4 & $1: 25$ & B cells & Central Laboratory of the Blood \\
CD19 & & $1: 200$ & p15E & Transfusion Service, Amsterdam \\
4F5 & & & & Kindly provided by G. J. Cianciolo \\
\hline
\end{tabular}

The first objective of this study is to give a detailed analysis of the pattern of tumor infiltration by macrophages, defined as large rounded cells strongly positive for CD68 and CD14 and by dendritic cells defined as stellateshaped cells positive for L25, RFD1 and S100. The spatial relationship between dendritic cells, macrophages and malignant epithelial cells and the spatial relationship of these cells to infiltrating $\mathrm{T}$ lymphocytes and B lymphocytes was studied as well.

Carcinoma cells, including head and neck squamous carcinoma cells are capable of producing factors that exert a variety of suppressive effects on different types of immune cells. Some of these tumor factors also demonstrate a suppressive effect on the chemotactic capability of monocytes/macrophages and the capability of dendritic cells to form clusters with allogeneic lymphocytes. The factors produced show a structural and functional homology with a retroviral transmembranal (TM) protein called p15E [5], hence the name p15E-like factors. It is not yet clear if any relationship exists between the degree of TM factor expression in head and neck cancer cells in vivo and the infiltration pattern of macrophages and dendritic cells in and around the malignant cells. We therefore also studied the relationship of the immunohistomorphological expression of this TM factor in head and neck squamous carcinoma cells and the pattern of infiltration of macrophages and dendritic cells.

\section{Materials and methods}

Patients and tissues. Samples of fresh tumor tissue were obtained from surgically removed tumors of 18 patients with squamous-cell carcinoma of the head and neck; 7 carcinomas of the oral cavity, 6 oropharyngeal carcinomas, 3 carcinomas of the hypopharynx and 2 of the larynx were included. Small representative tissue parts of the removed tumor were frozen in liquid nitrogen and stored at $-80^{\circ} \mathrm{C}$. Tissue was taken from the central solid part of the tumor to avoid the effect of superficial bacterial infection on lymphoid cell infiltration; signs of infection were not present in any of the tumor biopsies.

Immunohistology. Serial 6- $\mu \mathrm{m}$ cryostat sections were cut and mounted on slides, air-dried and fixed in acetone. Normal rabbit serum (Dakopatts, Copenhagen, Denmark) was added and gently removed by tapping the slides after $15 \mathrm{~min}$. The slides were thereafter incubated for $\mathrm{l} \mathrm{h}$ at room temperature with the monoclonal antibodies listed in Table 1, to identify the various infiltrating lymphoid cells. All antibodies were diluted in phosphate-buffered saline (PBS), pH 7.4 enriched with $1 \%$ bovine serum albumin (Sigma, St. Louis, Mo.) before incubation. After incubation the sections were gently rinsed with PBS to wash away excess monocional antibodies for at least $15 \mathrm{~min}$ and thereafter incubated with horseradish-peroxidase-conjugated rabbit anti-(mouse Ig) serum (Dakopatts, Copenhagen, Denmark) with $1 \%$ human pooled serum for 30 min. Subsequently, the sections were rinsed with PBS for another $15 \mathrm{~min}$ and stained for peroxidase activity with $3.3^{\prime}$-diaminobenzidine tetrahydrochloride (Sigma, St. Louis, Mo.) in PBS containing $0.01 \%$ $\mathrm{H}_{2} \mathrm{O}_{2}$. The sections were rinsed in tap water, counterstained with hematoxylin for $30 \mathrm{~s}$, dehydrated and mounted in malinol. All sections were additionally tested for acid phosphatase activity with naphthol AS-BI phosphatase as substrate and hexazotized pararosaniline as diazonium salt.

The slides were studied by two of the authors (J. K. and H. D.) using a Zeiss light microscope at various magnifications. Tumors were studied for morphology and lymphoid cell infiltration.

Cell infiltration was semiquantitavely scored as follows: - , no cells present; \pm , few scattered cells present; + , diffuse compartmental infiltration by solitary cells; ++, marked compartmental cell infiltration with no apparent organization; +++ , heavy cell accumulation with a clear infiltration architecture; ++++ , massive clotting of cells.

\section{Results}

Studying the immunohistological appearance of specimens of head and neck carcinoma, we could clearly make a distinction between areas composed of malignant squamous epithelial cells (parenchymal tumor nests) and areas of surrounding stromal connective tissue.

In the stromal connective tissue three types of lymphoid cell infiltration could be identified: (a) an infiltration by scattered and mostly single lymphoid cells. (b) small areas of densely packed lymphoid cells with a certain degree of organization and (c) small granuloma-like structures. The first type of stromal infiltration was the most predominant.

In the malignant epithelial areas (the tumor nests) there was also a scattered infiltration of single lymphoid cells.

\section{The infiltration pattern of scattered, mostly single lymphoid cells in the stromal compartment of the tumor (Table 2)}

Many CD68+ large cells were present, which morphologically varied from monocyte-like cells to very large, round, strongly staining macrophages. A similar pattern of distribution was seen for CD14+ cells. 
Table 2. Lymphoid cell infiltration in the stromal compartment and into malignant epithelial areas

\begin{tabular}{|c|c|c|c|c|}
\hline Cells & $\begin{array}{l}\text { Areas of } \\
\text { scattered } \\
\text { lymphoid cell } 1 \\
\text { infiltration }\end{array}$ & $\begin{array}{l}\text { Areas of dense } \\
\text { packed } \\
\text { lymphoid cell } \\
\text { accumulations }\end{array}$ & $\begin{array}{l}\text { Granulo- } \\
\text { matous } \\
\text { structures }\end{array}$ & $\begin{array}{l}\text { Cancer } \\
\text { nest } \\
\text { infilltra- } \\
\text { tion }\end{array}$ \\
\hline \multicolumn{5}{|l|}{ Dendritic cells } \\
\hline $\mathrm{S} 100$ & + & ++ & - & + \\
\hline CD1a & + & \pm & - & - \\
\hline RFD $1 / L 25$ & ++ & ++ & + & \pm to + \\
\hline \multicolumn{5}{|l|}{ Macrophages } \\
\hline CD68 & +++ & +++ (particularly in & ++++ & ++ \\
\hline CD14 & ++ & $++B$ cell areas) & ++ & + \\
\hline \multicolumn{5}{|l|}{ T cells } \\
\hline CD3 & + to ++ & +++ to ++++ & - & \pm \\
\hline \multicolumn{5}{|l|}{ B cells } \\
\hline CD19 & - & - to +++ & - & - \\
\hline
\end{tabular}

RFD1+ stellate-shaped cells were also clearly present, however, generally in fewer numbers than the $\mathrm{CD} 68^{+}$macrophages. The $\mathrm{RFDI}^{+}$dendritic cells were also positive for other MHC class II molecules (as identified with the mAb OKla); the cells were, however, less positive for L25 and markedly less positive for $\mathrm{CD} 1 \mathrm{a}$ and the $\mathrm{S} 100$ protein (though they were positive).

The number of scattered single $\mathrm{CD} 3+$ lymphocytes varied from tumor to tumor, there being no apparent relationship between $\mathrm{CD} 3+$ lymphocyte infiltration and the differentiation grade of the tumor. A large proportion of the small round $\mathrm{CD}^{3}+\mathrm{T}$ lymphocytes were also positive for MHC class II and RFD1. CD19+ B cells were not present in this type of infiltrate.

\section{Areas of dense, packed lymphoid cells in the stromal compartment (Table 2)}

Small areas of dense packed lymphoid cell infiltration were present in 8/18 of the studied tumor specimens (Fig. 1).
These dense lymphoid cell areas were randomly localized and not particularly located in the vicinity of, or at the borders of the epithelial tumor nests. Inter-tumoral variation was extensive, but intra-tumoral variation was limited with respect to the number of such areas.

The areas of dense lymphoid cells had a certain degree of architecture with identifiable areas of $\mathrm{CD}^{+} \mathrm{T}$ cell accumulation and, in an occasional tumor, a central area of CD19+ B cells. These latter areas are reminiscent of early $\mathrm{B}$ cell follicles. RFD1+ and $\mathrm{L} 25^{+}$stellate-shaped cells were strongly represented within the $\mathrm{CD}^{+} \mathrm{T}$ cell areas. In the S100 staining, the presence of dendritic cells within the $\mathrm{T}$ cell zones was also obvious; $\mathrm{CD}$ la positivity was, however, almost absent in these areas. With respect to the central B cell areas it was clear that in particular CD68+ and $\mathrm{CD} 14+$ monocytes/macrophages could be identified within these areas.

\section{Granulomatous structures within the stromal compartment (Table 2)}

Granulomatous structures were mostly small and always located near the borders of the malignant epithelial areas. They were present in $7 / 18$ of the specimens studied (Fig. 2). The presence or absence of these lymphoid cell structures was again not dependent on tumor differentiation, nor was there a relationship with the presence of the dense, packed lymphoid cell accumulations. The cells forming the granulomas were strongly positive for the macrophage marker CD68, while being only weakly positive for CD14, RFD1 and L25. T lymphocytes $\left(\mathrm{CD}^{+}\right)$and B lymphocytes (CD19+) were not present within these structures.

\section{Scattered infiltration of lymphoid cells in the parenchymal areas of malignant epithelial cells (Table 2)}

$\mathrm{CD} 8^{+}$macrophage infiltration was markedly present in most tumors, within the parenchymal areas of malignant epithelial cells, though, less than in the stromal areas

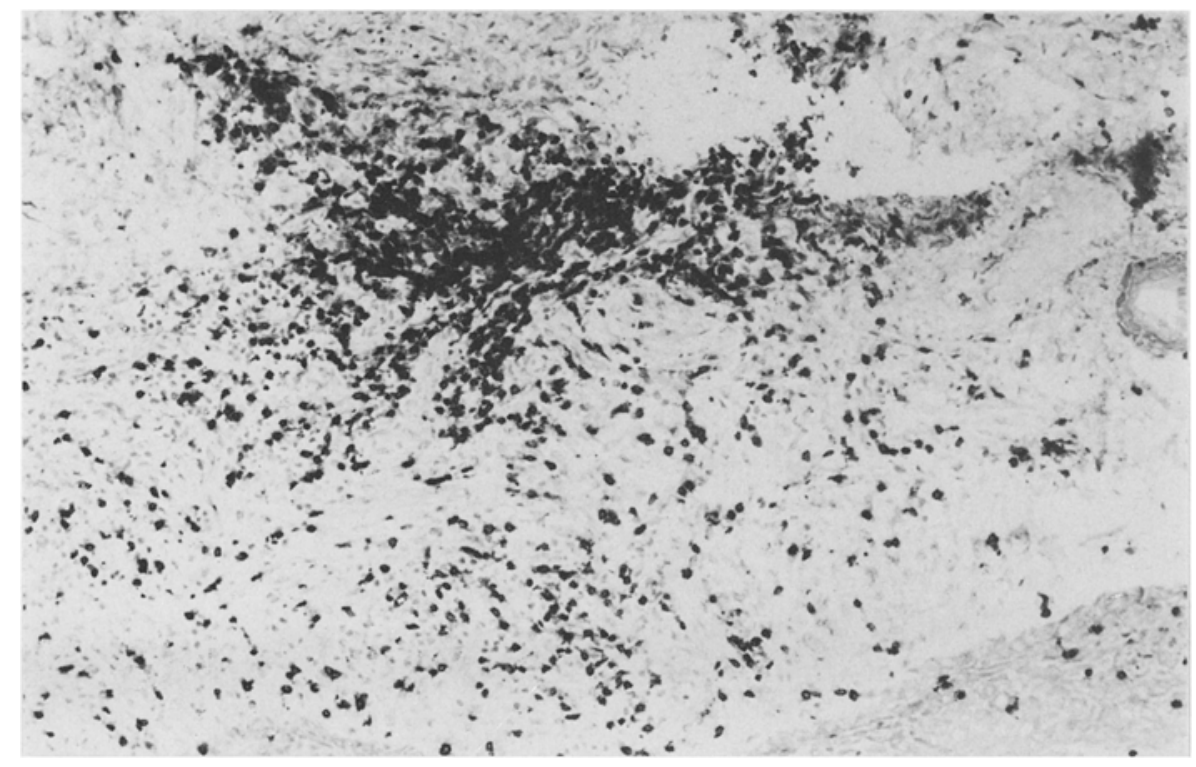

Fig. 1. Area of dense CD3+ lymphoid cell infiltration in stromal compartment. Magnification: $36 x$ 

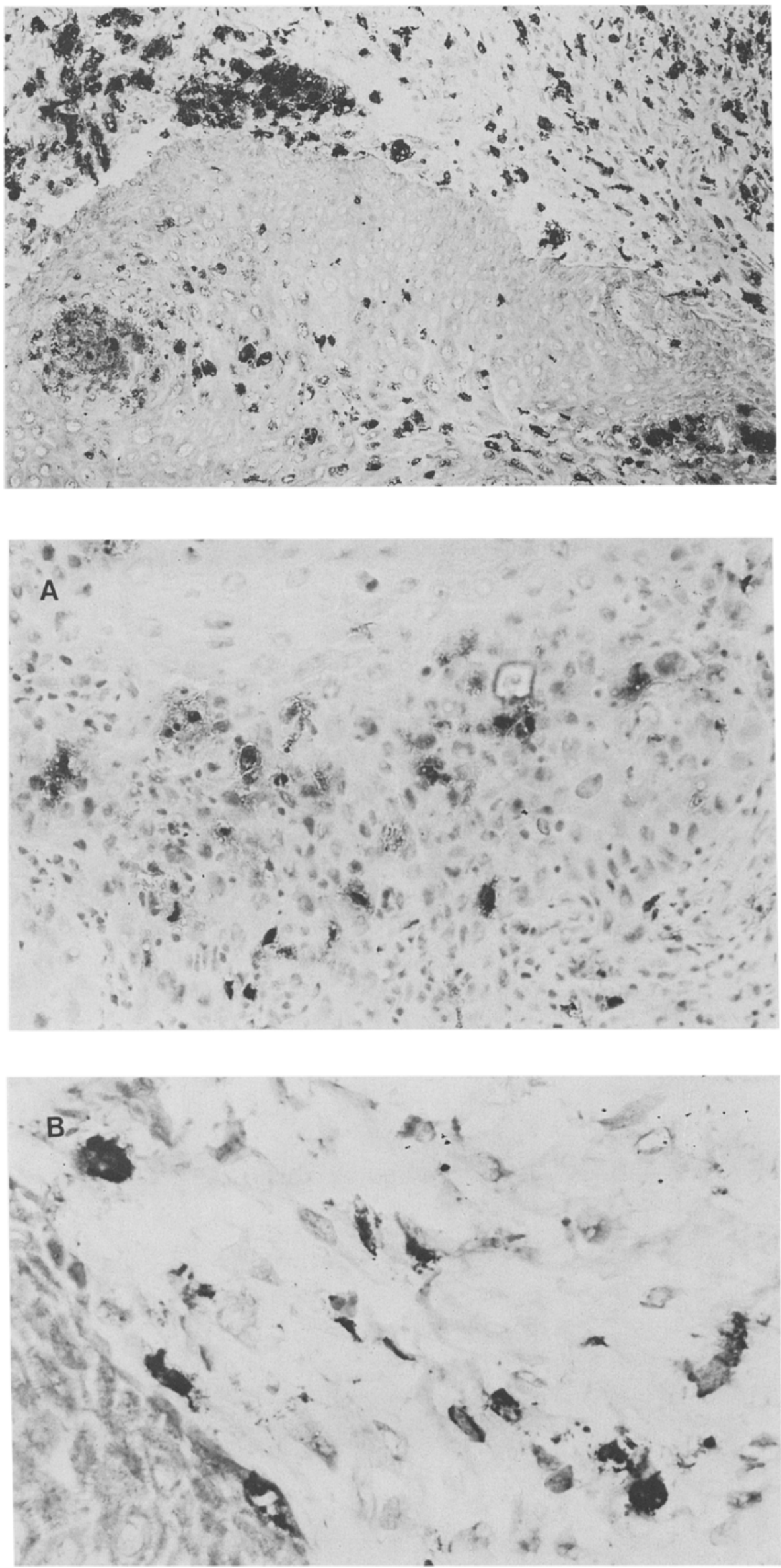

Fig. 2. Granulomatous $\mathrm{CD}^{2+}{ }^{+}$structure within the stromal compartment. Note also intratumoral $\mathrm{CD}^{2} 8^{+}$macrophage infiltration. Magnification: $36 \times$
Fig. 3. $\$ 100^{+}$dendritic cell infiltration into malignant epithelial area and surrounding stroma. Magnification: A $80 \times ;$ B $200 \times$ 
(Fig. 2). The pattern of infiltration between the different tumors, but also within an individual tumor was often variable. There was a stronger and more diffuse infiltration in poorly differentiated tumors, the $\mathrm{CD} 68^{+}$macrophages often reaching the central areas of the tumor nests. In well-differentiated tumor tissue the infiltration was less extensive, the CD68+ macrophages staying closer to the outer zones of the tumor. A similar pattern of CD14+ monocyte/macrophage infiltration was found although numbers of such monocytes/macrophages were considerably lower. Very few CD1 $\mathrm{a}^{+}, \mathrm{RFD} 1^{+}$and $\mathrm{L} 25^{+}$cells with a classic dendritic cell morphology were present in welldifferentiated malignant epithelial areas; however, in poorly differentiated tumors more RFD1+ cells were identifiable. The few infiltrating dendritic cells in the welldifferentiated tumors stayed at the borders of the tumor nests. Intra-epithelial infiltration of $\mathrm{S} 100^{+}$dendritic cells was considerable and comparable to stromal $\mathrm{S} 100^{+}$dendritic cell infiltration (Fig. $3 \mathrm{~A}, \mathrm{~B}$ ).

$\mathrm{CD} 3+$ lymphocytes also basically infiltrated the outer zones of the tumor nests (similar to the CD1 a ${ }^{+}, \mathrm{RFD}^{+}$and $\mathrm{L}_{25^{+}}$dendritic cells in the well-differentiated tumors), again in considerably lower numbers compared to their infiltration into the stromal compartment. Infiltration of $\mathrm{CD} 3+$ lymphocytes into poorly differentiated tumors was more extensive than into well-differentiated tumors. CD19+ B cells were virtually absent from these malignant epithelial areas.

In all tumors, the malignant epithelial structures stained clearly positive for the anti-p15E antibody 4F5 to an extent that mostly depended on the differentiation grade of the tumor. Expression varied from weak to strong between tumors and sometimes even within tumors. There was, however, no relationship between the intensity of p15E expression and the severity of lymphoid cell infiltration of any kind.

\section{Discussion}

The dendritic cells in the antigen-presenting accessory cell par excellence $[2,10,11]$. The cell exposes on its long cytoplasmic extensions a high density of MHC class I and II molecules (the antigen-presenting molecules), and actively seeks contact with surrounding cells including lymphocytes to present the antigens. During this antigen presentation the cell forms cell clusters with the lymphocytes. Apart from this cluster formation, in which a micro-environment suitable for antigen presentation is created, the dendritic cell is capable of producing a series of cytokines that activate $\mathrm{T}$ and $\mathrm{B}$ lymphocytes. Dendritic cells do not only occur in the $\mathrm{T}$ cell areas of spleen and lymph nodes as so-called interdigitating dendritic cells, but also occur as the Langerhans cells in the epidermis and dermis of the skin and mucosal surfaces. It is now generally accepted that the dendritic cells of the skin and mucosal surfaces travel via the lymph to the draining lymph nodes, transporting antigens from the periphery to the lymphoid organs to present the antigens to lymphocytes to initiate immune reactivity in the lymphoid spleen [12].
Although no specific $\mathrm{mAb}$ for the staining of dendritic cells exists, RFD1 and L25 can be used adequately (together with classical morphological characteristics such as shape) to identify the lymphoid dendritic cells present in the T cell areas of spleen and lymph nodes. S100 and CD1 are reasonably characteristic for the Langerhans cells of the skin (these marker proteins are also present in some dendritic cells of the lymphoid tissues).

In this study we found that RFD1+ and $\mathrm{L}^{2} 5^{+}$dendritic cells were markedly present within the $\mathrm{T}$ cell areas of lymphoid cell accumulations present in the stromal compartment between tumor nests. These dendritic cells were virtually negative for the markers S100 and CD1. The position of these lymphoid dendritic cells indicates their antigen-presenting function and suggests an active stimulation of the immune system in the dense lymphoid areas in at least some tumors. RFD1 ${ }^{+}$and $\mathrm{L} 25^{+}$dendritic cells were also diffusely present in the stromal compartment, but only scarcely within the well-differentiated malignant epithelial areas. The intra-epithelial infiltration of $\mathrm{S} 100^{+}$dendritic cells was considerable and comparable to stromal $\mathrm{SlO0}^{+}$ dendritic cell infiltration. This observation is in agreement with the classical notion that intra-epithelial dendritic cells, often referred to as Langerhans cells, are mainly S100-positive, and our data thus suggest a more Langerhans-celllike characteristic of the dendritic cells that have infiltrated the malignant epithelial cell areas.

Our observations are hence by and large in agreement with the study of Nomori et al. [16] on nasopharyngeal carcinoma. These authors found $\mathrm{S} 100^{+}$dendritic cells primarily located within the tumor nests, and found few of these cells in the surrounding connective tissue. Similar findings have been reported by Nakano et al. [14] in squamous-cell carcinoma of the uterine cervix; they described that the $\mathrm{S} 100^{+}$dendritic cells were mainly located in between the malignant epithelial tumor cells. In gastric adenocarcinoma Tsujitani et al. [20,21] described clusters of $\mathrm{S} 100^{+}$dendritic cells in the tumor stroma as well as an infiltration in between the cancer cells. In all these studies S100 only was used to identify dendritic cells, and it is therefore difficult to draw any conclusions concerning the dendritic cells that are negative for this protein (the majority of the dendritic cells present in $T$ cell areas of the focal lymphocyte infiltrates found in our study). It is of importance to note that all three of the above-mentioned authors correlated a marked dendritic cell infiltration with a better prognosis. These findings indicate that dendritic cells probably play a more important role in the immune defense against cancer than do scavenger macrophages. The number of patients and length of follow-up period in our study are so far insufficient for correlation studies, but later reports will address this issue.

Macrophages with a scavenger and cytotoxic function are mainly involved in the efferent arm of the immune response. Such cells are markedly positive for CD68 and lysosomal enzymes such as acid phosphatase. In our study $\mathrm{CD} 8^{+}$macrophages were strongly represented both within the connective tissue compartment as well as in the malignant epithelial areas; however, the infiltration into the latter area varied widely between the various tumors, some showing hardly any, others showing massive intra-epitheli- 
al invasion. The latter pattern may indicate a stronger cytotoxic response to these tumors; however, histologically clear tumor cell destruction was not seen.

Ki-M7 (a monoclonal antibody that reacts with CD68, used in our study) stains mature CD68+ macrophages [7]. Despite the fact that macrophages have in vivo and in vitro a cytotoxic/cytolytic effect when activated by lymphokines or bacterial products, their precise role in tumor immunology in vivo remains uncertain. Cameron and Stromberg [4] found a defect in tumoricidal capacity in head and neck cancer; half of the patients in their study possessed macrophages that were non-cytotoxic toward tumor cells in vitro. Allen [1] found no histological evidence for a cytotoxic role of macrophages in colorectal tumors. In murine sarcomas and carcinomas, tumor-associated macrophages were even thought to stimulate tumor growth [13]. It has been suggested [17] that tumor cells may become insensitive to cytolysis in vivo by activated macrophages by building up a resistance to tumor necrosis factors, which are mediators in the lymphokine activation of macrophages.

Neuchrist et al. [15], also using immunohistology, showed that most tumor-infiltrating macrophages are of a functionally mature phenotype. The distribution these authors described is similar to our findings, with an extensive stromal macrophage invasion and some infiltration into the areas of the malignant epithelial cells (with a wide inter-tumoral variation). These authors, however, did find (in contrast to us) a correlation between the extent of $\mathrm{CD}^{+}$ lymphocyte infiltration and the infiltration by macrophages. Others have also stressed the important role of T-cell-activating macrophages in direct tumor defence [23]. It must be noted, however, that Neuchrist et al. used markers such as a-HLA-DR, a-Fcy receptors I, II, III, and $\mathrm{Rm} 3 / 1$ to identify the macrophages. We consider HLA$\mathrm{DR}^{+}$cells not as classical macrophages but, when stellateshaped, as dendritic cells; this might explain the discrepancy between our results and those of others. We did find a relationship between the infiltration of HLA-DR+ dendritic cells and T cells.

In nasopharyngeal carcinoma Nomori [16] found that most lysozyme-antibody-stained macrophages surrounded the tumor nests although he did not describe the granulomatous structures of our study. In the granulomatous structures present in the stromal compartment close to the tumor nests all cells were strongly positive for CD68, in fact the histology suggests to us that these areas were almost predominantly composed of large, fused macrophages supporting the classically held view that granuloma's are mainly involved in the degradation of persistent antigens or non-degradable materials. A marked intra-epithelial infiltration of macrophages in adenocarcinoma of the stomach and in breast cancer was found by Tsujitani et al. [20,21] and Zuk et al. [24]. We also found a marked infiltration with such cells mainly in poorly differentiated head and neck squamous-cell carcinomas.

Cianciolo [6] demonstrated in 1980 the presence of a low-molecular-mass (low- $M_{\mathrm{r}}$ ) factor in both animal as well as human cancers. This factor had an inhibitory effect on monocyte chemotaxis and appeared later to be absorbable by any of three different mAb to a retroviral capsular protein, namely p15E. Tan [18] showed that the p15E-like low- $M_{\mathrm{r}}$ factors were also present in the tumors of patients with head and neck cancer as well as in the serum of these patients. The p15E-like factors, presently called TM factors, have a suppressive effect not only on monocyte chemotaxis but also on the function of various cells of the immune system. The factors inhibit $\mathrm{IL}-2$ dependent $\mathrm{T}$ cell proliferation, they inhibit the blastogenic responses to mitogens and antigens, they suppress the natural killer cell activity, they hamper the clustering capacity of dendritic cells and they suppress the respiratory burst of human monocytes. All tumors in our study showed positivity for the anti-TM-factor mAb 4F5. However, no relationship between $\mathrm{p} 15 \mathrm{E}$ expression and any particular infiltrative pattern could be demonstrated. In our earlier report we were able to correlate tumor infiltration with chemotactic ability [3]. Important to note is that positive staining of cancer cells for anti-TM-factor mAb does not necessarily mean that the tumor produces or secretes these factors. Furthermore, recent work in our group [19] has suggested that the anti-TM-factor $\mathrm{mAb}$ may not be as specific for p15E as was believed earlier: it has been shown that these $\mathrm{mAb}$ cross-react with interferon $\alpha$ and possibly other cytokines. Future work will address this matter.

\section{References}

1. Allen C, Hogg N (1987) Elevation of infiltrating mononuclear phagocytes in human colorectal tumors. J Natl Cancer Inst 78: 465

2. Austyn JM (1987) Lymphoid dendritic cells. Immunology 62: 161

3. Balm AJM, Drexhage HA, von Blomberg M, Weltevreden EF, Veldhuizen RW, Mullink R, Snow GB (1984) Mononuclear phagocyte function in head and neck cancer. Chemotactic responsiveness of blood monocytes in correlation between histologic grade of the tumor and infiltration of these cells into the tumor area. Cancer 54: 1010

4. Cameron DJ, Stromberg BV (1984) The ability of macrophages from head and neck cancer patients to kill tumor cells. Effect of prostaglandin inhibitors in cytotoxicity. Cancer 54: 2403

5. Cianciolo GJ (1986) Antiinflammatory proteins associated with human and murine neoplasms. Biochim Biophys Acta 865: 69

6. Cianciolo GJ, Herberman RB, Snyderman R (1980) Depression of murine macrophage accululation by low molecular weight factors derived from spontaneous mammary carcinoma. J Natl Cancer Inst 65: 829

7. van Dongen JJM, Adriaansen HJ, Hooijkaas H (1985) Immunologic markers and their CD codes. J Immunol Methods 80: 1

8. Garraud O, Faucher A, Legrand E (1988) Impairment of monocyte functions in advanced head and neck cancer. Immunol Lett 18: 213

9. Guo M, Rabi BS, Johnson JT, Paradis IL (1987) Lymphocyte phenotypes at tumor margins in patients with head and neck cancer. Head Neck Surg? 265

10. Gyure LA, Barfoot R, Denkan S, Hall JG (1987) Immunity to a syngeneic sarcoma induced in rats by dendritic lymph cells exposed to tumor either in vivo or in vitro. Br J Cancer 55: 17

11. Inoba K, Witmer MD, Steinman RM (1984) Clustering of dendritic cells, helper T lymphocytes and histocompatible B cells during primary antibody responses in vitro. J Exp Med 160: 858

12. Knight SC, Bedford P, Hunt R (1985) The role of dendritic cells in the initiation of immune responses to contact sensitizers. $\Pi \mathrm{L}$ studies in nude mice. Cell Immunol 94: 435

13. Milas L, Wike J, Hunter N, Volpe J, Basic I (1987) Macrophage content of murine sarcomas and carcinomas: associations with tumor growth parameters and tumor radiocurability. Cancer Res 47: 1069 
14. Nakano T, Oka K, Arai T, Morita S, Tsunemoto H (1989) Prognostic significance of Langerhans' cell infiltration in radiation therapy for squamous cell carcinoma of the uterine cervix. Arch Pathol Lab med 113: 507

15. Neuchrist C, Grasl M, Scheiner O, Lassmann H, Ehrenberger K, Kraft D (1990) Squamous cell carcinoma: infiltrating monocyte/macrophage subpopulations express functional mature phenotype. Br J Cancer 62: 748

16. Nomori H, Watanabe $S$, Nakajima T, Shimosato $Y$, Kameya $T$ (1986) Histiocytes in nasopharyngeal carcinoma in relation to prognosis. Cancer 57: 100

17. Remels L, Fransen L, Huygen K, De Boetselier P (1988) Immunological aspects of tumor-macrophage interactions. Adv Exp Med Biol 233: 49

18. Tan IB, Drexhage HA, Mullink R, Hensen-Logmans S, De HaanMeulman M, Snow GB, Balm AJM (1987) Immunohistochemical detection of retroviral-p15E-related material in carcinomas of the head and neck. Otolaryngol Head Neck Surg 96: 251

19. Tas MPR, Simons PJ, Balm FJM, Drexhage HA (1993) Depressed monocyte polarization and clustering of dendritic cells in patients with head and neck cancer: in vitro restoration of this immunosuppression by thymic hormones. Cancer Immunol Immunother 36: 108

20. Tsujitani S, Furukawa T, Tamada R, Okamura T, Yasumoto $\mathrm{K}$, Sugimachi K (1987) Langerhans cells and prognosis in patients with gastric carcinoma. Cancer 59: 501

21. Tsujitani S, Kakeji Y, Watanabe A, Kohnoe S, Maehara Y, Sugimachi K (1990) Infiltration of dendritic cells in relation to tumor invasion and lymph node metastasis in human gastric cancer. Cancer 66: 2012

22. Wolf GT, Hudson J, Peterson KA, Miller HL, McLatchey KD (1986) Otolaryngol Head Neck Surg 95: 142

23. Yoshida K, Tachibana T (1993) Prevention of lymph node metastases by adoptive transfer of CD4+ $\mathrm{T}$ lymphocytes admixed with irradiated tumor cells. Cancer Immunol Immunother 36: 323

24. Zuk JA, Walker RA (1987) Immunohistochemical analysis of HLA antigens and mononuclear infiltrates of benign and malignant breast. J Pathol 152: 275 